\title{
Histological appearances of the levator palpebrae superioris muscle in the Marcus Gunn phenomenon
}

\author{
R W LYNESS,' J R O COLLIN, ${ }^{2} R$ A ALEXANDER, ${ }^{2}$ AND A GARNER ${ }^{2}$ \\ From the Departments of 'Pathology and Ophthalmology, the Queen's University of Belfast, Royal Victoria \\ Hospital, Belfast, and the ${ }^{2}$ Institute of Ophthalmology, University of London, 17-25 Cayton Street, \\ London ECIV $9 A T$
}

SUMMARY The levator palpebrae superioris muscles from 12 cases of Marcus Gunn phenomenon were examined histologically by means of histochemistry and morphometry. The appearances were of a neurogenic atrophy with pathological changes occurring in both the affected side and the clinically normal side, suggesting that the causative lesion was located in the central nervous system.

In 1883 Marcus Gunn described a case of unilateral congenital ptosis affecting the left side.' The salient features of the original case were contraction of the levator palpebrae muscle in association with the external pterygoid muscle, a diminished action of the levator muscle (tonic and active) in its association with other muscles supplied by the third nerve, an imperfect relaxation of the levator muscle when the eyelids were gently closed, and a diminished size of pupil on the affected side. The pupillary movements were described as being normal with no evidence of a Horner's syndrome.

The committee ${ }^{1}$ that reviewed the findings of Marcus Gunn's case postulated the existence of abnormal connections between the third and fifth cranial nerve motor nuclei. An electromyographic study $^{2}$ of one elderly male suffering from the Marcus Gunn phenomenon (MGP) suggested that the dysfunction was located in the mid brain and brain stem. It has been suggested ${ }^{3}$ that the ptosis and abnormal movements in the MGP are analogous to the movements seen in fish-namely, the co-ordinated jaw and spiracle musculature. An alternative explanation $^{4}$ is that the Marcus Gunn phenomenon may represent a supranuclear failure to suppress a primitive reflex.

Several studies of the morphology in congenital ptosis from 1890 to 1955 were reviewed by Berke and Wadsworth. ${ }^{5}$ A range of gross and histological abnormalities of the levator muscle, often in association with abnormalities of the superior rectus muscle, Correspondence to Professor A Garner. were noted. A study of a case of left sided ptosis by Siemerling ${ }^{6}$ showed degeneration of the levator muscle, the third cranial nerve, and nuclei on the

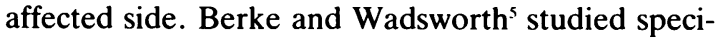
mens from the levator muscles of patients undergoing surgery for the correction of ptosis. They found that in congenital ptosis the number of striated muscle fibres in their specimens was inversely related to the severity of the ptosis and extent of eyelid movement (levator function). In patients with Marcus Gunn phenomenon Müller's muscle and striated levator muscle fibres were present, but no comment was made on the quality of the levator muscle in these cases.

We describe the histopathological appearances of levator muscles removed from 12 patients undergoing surgery (bilateral brow suspension) to correct a ptosis due to the Marcus Gunn phenomenon.

\section{Materials and methods}

In 12 patients with ptosis and the Marcus Gunn phenomenon the levator muscles on both sides were isolated via a posterior approach. Each muscle was transected above the origin of Müller's muscle and a segment of the whole striated muscle varying in length from 3 to $10 \mathrm{~mm}$ was excised and placed in separate bottles containing potassium citrate and magnesium sulphate buffered ammonium sulphate fixative. ' The eyelids were then suspended from the frontalis muscle with strips of autogenous fascia lata to correct the ptosis (bilateral brow suspension). 
The biopsy, specimens thus obtained were rehydrated in the potassium citrate and magnesium buffer without ammonium sulphate, then snap frozen in nitrogen cooled isopentane. Cryostat sections were prepared and stained by the following methods: (1) haematoxylin and eosin; (2) Gomori trichrome (one step) method; ${ }^{8}$ (3) ATP (myosin adenosine triphosphatase) at $\mathrm{pH} 4 \cdot 3,4 \cdot 6$, and $10 \cdot 4 ;^{411}$ (4) NADHTR (nicotinic adenine dinucleotide (reduced) tetrazolium reductase); ${ }^{\prime \prime}(5)$ non-specific acid phosphatase $;^{12}(6)$ acetylcholinesterase. ${ }^{13}$.

The histological sections were examined by light microscopy. The diameter of individual muscle fibres and the numbers of muscle fibres were measured with eyepiece graticules. The fibre diameters of $150-200$ of each of the two main muscle fibre types (type 1 or slow fibre and type 2 or fast fibre) were measured. Mean values with standard deviations for both fibre

Table 1 Clinical data

\begin{tabular}{llll}
\hline Case & Sex & Affected side & Ageat operation \\
\hline 1 & F & Right & $41 / 2$ years \\
2 & F & Left & 4 years \\
3 & M & Right & $61 / 2$ years \\
4 & M & Right & 7 years \\
5 & M & Right & 17 years \\
6 & F & Left & $81 / 2$ years \\
7 & M & Left & $43 / 4$ years \\
8 & M & Left & $71 / 2$ years \\
9 & M & Right & $73 / 4$ years \\
10 & F & Left & 5 years \\
11 & M & Left & 5 years \\
12 & F & Right & $73 / 4$ years \\
& & & Age at necropsy \\
Control I (cystic & M & - & 15 years \\
fibrosis) & & & 10 years \\
Control II (road & F & - & \\
traffic accident) & & & \\
\hline
\end{tabular}

Table 2 Muscle Fibre Density (fibres/sq mm)

\begin{tabular}{llc}
\hline Case & Affected side & Clinically normal side \\
\hline 1 & 182 & 192 \\
2 & 356 & 1671 \\
3 & 467 & 525 \\
4 & 478 & 537 \\
5 & 131 & 202 \\
6 & 242 & 352 \\
7 & 788 & 892 \\
8 & 425 & 776 \\
9 & 154 & 278 \\
10 & 578 & 1638 \\
11 & 183 & 462 \\
12 & 252 & 285 \\
& Right side & Left side \\
Control I & 1200 & 1265 \\
Control II & 1541 & 1626 \\
\hline
\end{tabular}

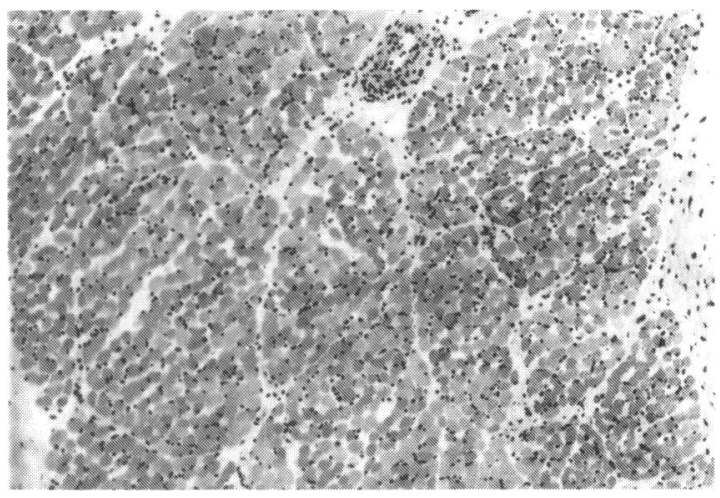

Fig. 1 Histological section of levator muscle from a normal control subject showing a normal density of uniformly sized muscle fibres arranged in fascicles. $H$ and $E$.

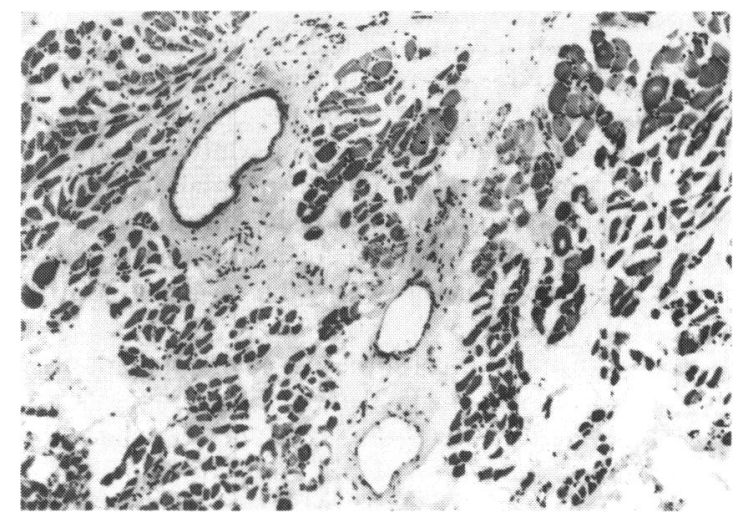

Fig. 2 Histological section of levator muscle from an affected side (MGP) showing a marked decrease in the muscle fibre density and variation in the size of the remaining muscle fibres. $H$ and $E$.

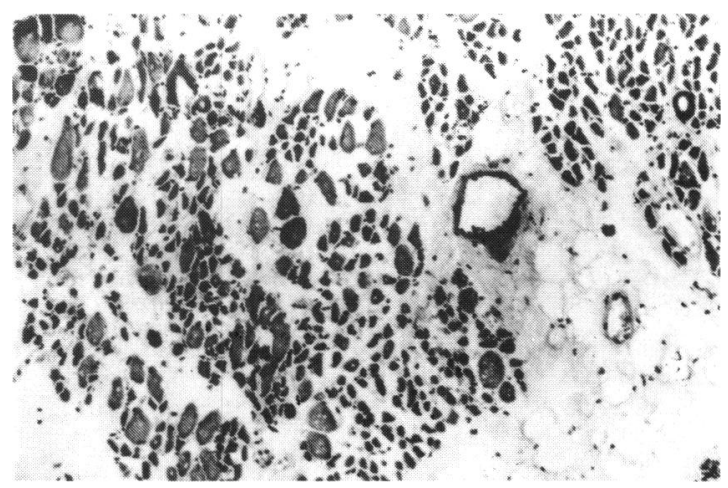

Fig. 3 Histological section of levator muscle from a clinically normal side (MGP) showing a decrease in fibre density and variation in the size of the remaining muscle fibres. $H$ and $E$. 
types and an overall value for the biopsy were calculated.

Levator palpebrae superioris muscles were obtained from two necropsy cases to act as 'normal' controls. The necropsy subjects were aged 15 years and 10 years respectively and died of cystic fibrosis and as a result of a road traffic accident. Neither case had any history of oculomotor problems or ophthalmic disease. Specimens obtained from these two cases were processed as above.

\section{Results}

The mean age at surgery was 7 years (Table 1). Of the 12 cases in the series seven $(58 \%)$ were male and five $(42 \%)$ female. Both sides were affected equally.

All biopsies contained striated muscle fibres. The density of the muscle fibres in the levator muscles of the control subjects was roughly equal on both sides (Table 2). The density of muscle fibres was reduced on the affected side in all cases of MGP when compared with the biopsy from the clinically normal side. In some the density was only marginally reduced from that of the clinically normal side. There was an impression that the density of muscle fibres from both sides of the patients suffering the MGP was reduced in comparison with the normal control subjects (Figs. 1, 2, 3).

In the normal control subjects the mean diameters (Table 3 ) for type 1, type 2, and overall muscle fibres were in the region of $18-19.5 \mu \mathrm{m}$, and the values for the standard deviation were approximately 3.75. Comparison of the pairs of muscles obtained from each normal control subject showed a slight variation in the values.
A wide range of values was obtained for the type 1 , type 2 , and overall mean fibre diameters in the various biopsies from the clinically normal sides. Comparison of the clinically normal biopsies with the paired biopsy from the affected contralateral levator muscle showed that there was a general tendency to hypertrophy of the remaining muscle fibres on the affected side (type 2 fibres more so than type 1 fibres), though some pairs of biopsies showed little difference in the values for the mean fibre diameters. Comparison of the standard deviations and histograms (Figs. 4, 5) of the two sides showed a generally wide range of values for the fibre diameters of the muscle fibres from the affected side.

Comparison of the values and histograms obtained from the normal control subjects with the clinically normal levator muscles from the affected subjects showed that there was a greater variation in the muscle fibre diameters of some of the clinically normal levator muscles (range of means 11.75-37.5 $\mu \mathrm{m})$ than seen in the normal control levator muscles (range of means 18-19.76 $\mu \mathrm{m}$ ). It would also appear that there was a tendency to overall atrophy in both affected and clinically normal sides in comparison with the clinically normal control subjects.

The distribution of type 1 and type 2 fibres in the normal control muscle gave rise to a speckled pattern (Fig. 6). The ratio of the numbers of type 1 and type 2 fibres was approximately $1: 5$ in all four control levator muscles and in most areas of the muscles from the patients suffering from the Marcus Gunn phenomenon. However, there was muscle fibre type grouping (with associated hypertrophy or atrophy of the grouped fibres) in many of the specimens (Fig. 7) from the affected sides in cases of Marcus Gunn

Table 3 Muscle fibre diameters $(\mu \mathrm{m})$

\begin{tabular}{|c|c|c|c|c|c|c|c|}
\hline \multirow[t]{2}{*}{ Case } & \multirow[t]{2}{*}{ Age } & \multicolumn{3}{|l|}{ Affected side } & \multicolumn{3}{|c|}{ Clinically normal side } \\
\hline & & Type 1 & Type 2 & Overall & Type 1 & Type 2 & Overall \\
\hline 1 & $41 / 2$ & Not done & Not done & $21 \quad(8 \cdot 75)$ & Not done & Not done & $(5 \cdot 75)$ \\
\hline 2 & 4 & Not done & Not done & $(8 \cdot 5)$ & Not done & Not done & $(9 \cdot 25)$ \\
\hline 3 & $61 / 2$ & $17 \cdot 5(7)$ & $21 \quad(7 \cdot 5)$ & $19 \cdot 75(10)$ & $12 \cdot 25(6 \cdot 0)$ & $15 \cdot 5(7 \cdot 5)$ & (7) \\
\hline 4 & 7 & $15 \quad(6)$ & $20 \cdot 75(7 \cdot 5)$ & $17 \cdot 25(7 \cdot 25)$ & $16 \quad(6.0)$ & 20 & $18 \cdot 5(7 \cdot 5)$ \\
\hline 6 & $81 / 2$ & $19 \quad(6 \cdot 75)$ & $22.5(10 \cdot 5)$ & $20 \cdot 75(9 \cdot 0)$ & $15 \cdot 75(4 \cdot 75)$ & $19 \cdot 75(2 \cdot 4)$ & $17.5(5 \cdot 75)$ \\
\hline 7 & $43 / 4$ & $12 \quad(5 \cdot 5)$ & $14 \cdot 75(6 \cdot 5)$ & $13 \cdot 75(6 \cdot 25)$ & $10.5(3.25)$ & $13 \cdot 5(4 \cdot 25)$ & $12 \cdot 0$ (4) \\
\hline 8 & $71 / 2$ & $15 \cdot 75(5 \cdot 75)$ & $20 \cdot 75(7 \cdot 25)$ & $18 \cdot 25(7 \cdot 0)$ & $13 \cdot 25(5 \cdot 25)$ & $16 \cdot 9(6 \cdot 25)$ & $14 \cdot 75(6 \cdot 0)$ \\
\hline 9 & $73 / 4$ & Not done & Not done & $17 \cdot 75(7 \cdot 75)$ & Not done & Not done & $22.50(11 \cdot 5)$ \\
\hline 10 & 5 & $11 \cdot 5(7 \cdot 5)$ & $12 \cdot 25(5 \cdot 0)$ & $11 \cdot 75(6 \cdot 75)$ & $12 \cdot 75(5 \cdot 5)$ & $11 \cdot 25(4 \cdot 25)$ & $11 \cdot 75(4 \cdot 75)$ \\
\hline 11 & 5 & $17 \cdot 25(4 \cdot 75)$ & $21 \quad(7 \cdot 0)$ & $19 \cdot 5(6 \cdot 25)$ & $13 \cdot 5(4 \cdot 25)$ & $13 \cdot 25(5 \cdot 5)$ & $13 \cdot 25(5 \cdot 0)$ \\
\hline \multirow[t]{2}{*}{12} & $73 / 4$ & $13 \cdot 5(4 \cdot 5)$ & $19 \cdot 25(8 \cdot 5)$ & $16 \cdot 75(9)$ & $12 \cdot 25(5 \cdot 25)$ & $16.5(7.5)$ & $14 \cdot 75(7 \cdot 0)$ \\
\hline & & \multicolumn{3}{|l|}{ Right side } & \multicolumn{3}{|l|}{ Left side } \\
\hline Control I & $15 \mathrm{yr}$ & $19 \quad(3 \cdot 75)$ & $19 \cdot 75(3 \cdot 75)$ & $19 \cdot 5(3.75)$ & $18 \cdot 75(3 \cdot 25)$ & $19 \cdot 0(4 \cdot 25)$ & $19.0(3.75)$ \\
\hline Control II & $10 \mathrm{yr}$ & $18 \cdot 5(3.75)$ & $19.25(3.75)$ & $18 \cdot 75(3.75)$ & $17 \cdot 75(4 \cdot 5)$ & $18 \cdot 5(4 \cdot 75)$ & $18 \cdot 0(4 \cdot 75)$ \\
\hline
\end{tabular}

SD shown in parentheses. 
NORMAL CONTROL SIDE 1.

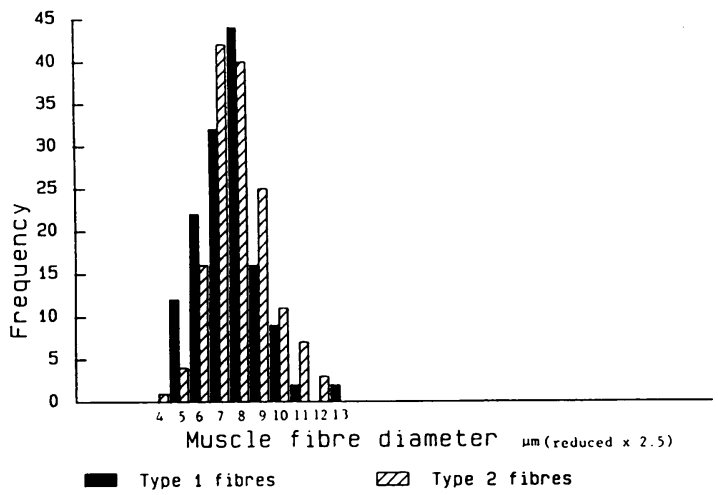

NORMAL CONTROL SIDE 2.

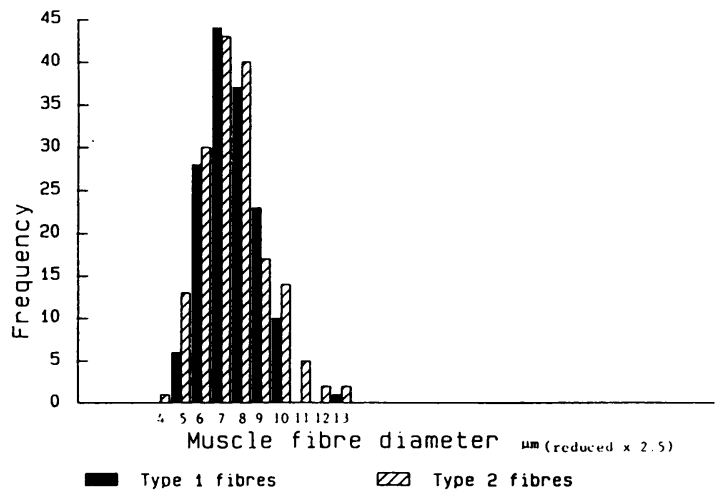

Fig. 4 Histograms showing the distribution of type 1 and type 2 muscle fibre diameters in levator muscles from a normal control subject.

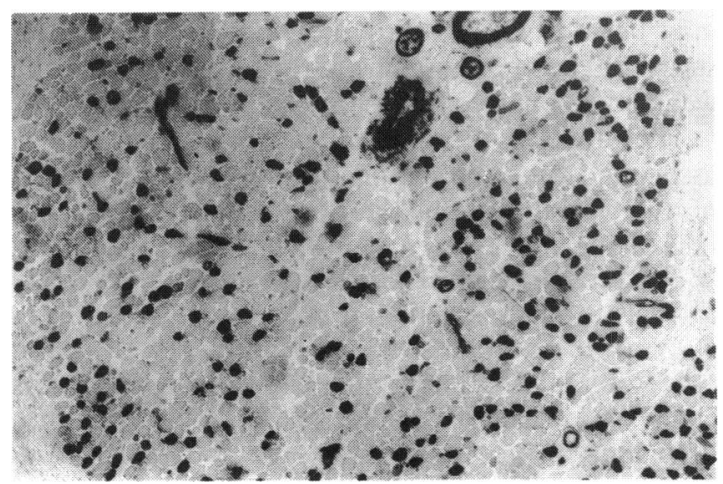

Fig. 6 ATPase preincubated at pH 4·3. Histological section of levator muscle from a normal control subject showing the same area as Fig. 1. Type 1 fibres are black and type 2 are pale grey. Note the speckled pattern.
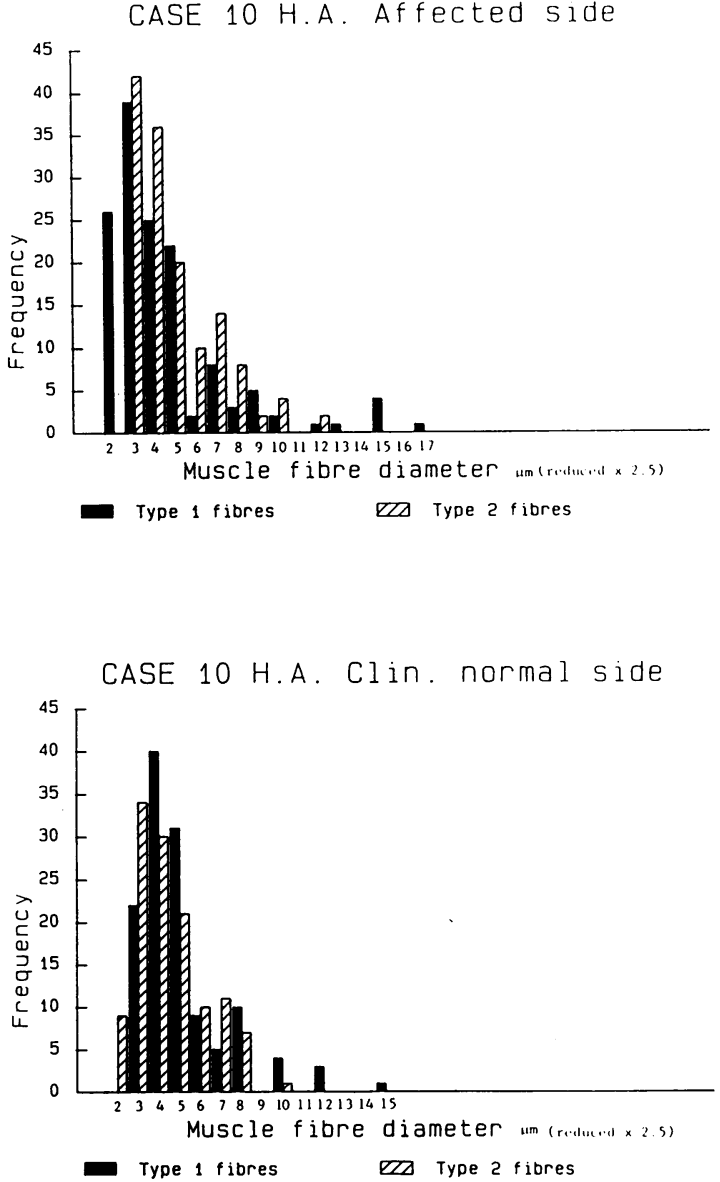

Fig. 5 Histograms showing the distribution of type 1 and type 2 muscle fibre diameters in a subject affected by the Marcus Gunn phenomenon. Note the widening of the distribution of fibre diameters and the overall shift to the left indicating generalised atrophy, more obvious on the affected side but present also on the clinically normal side.

phenomenon. In some of the specimens from the clinically normal side there were some small areas of fibre type grouping.

Histological sections from both sides of patients affected by MGP showed central accumulation of mitochondria within individual muscle fibres (Fig. 8). This change from the normal granular pattern of mitochondria seen in fibres from the normal control levator muscles (Fig. 9) was seen in small groups of muscle fibres scattered throughout the biopsies from both the affected and clinically normal sides.

Acid phosphatase staining showed no evidence of any lysosomal accumulations within muscle fibres or the presence of macrophages in the perimysium. No evidence of any synapses was seen in any of the biopsies with acetylcholinesterase. 


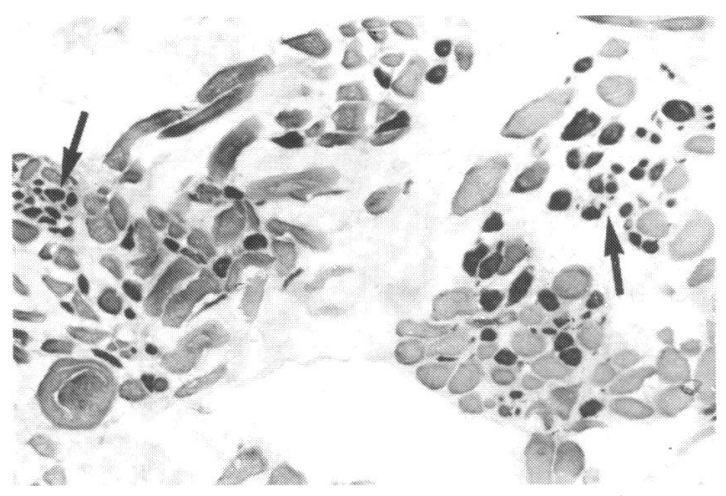

Fig. 7 ATPase preincubated at pH 4-3. Histological section of levator muscle from an affected side (MGP) showing a loss of the normal speckled pattern, with grouping (arrowed) of the type 1 muscle fibres (stained black) and the wide variation in the muscle fibre diameter.

\section{Discussion}

The site of our muscle biopsies from the distal. end of the levator muscle close to the origin of the aponeurosis and the use of a fixative solution in processing combined to make them difficult to interpret. However, it has been possible to identify trends in the appearances and, indeed, definite lesions in the 24 biopsies, especially when they were compared with similar material obtained from two cadavers. Using an ammonium sulphate fixative required that the specimens were rehydrated prior to freezing. This needed careful attention so as to minimise distortion of the morphology of the small biopsy specimens. The use of a fixative was justified by the need to transport the specimens from one

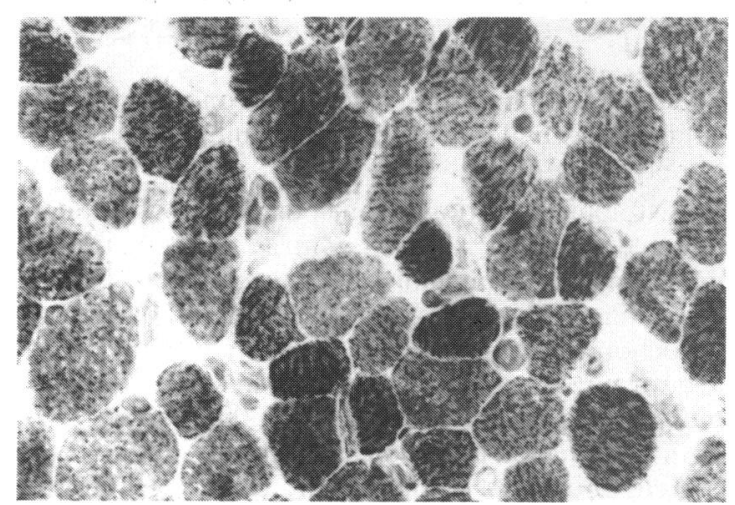

Fig. 9 Histological section of levator muscle from a normal control subject showing the normal granular distribution of mitochondria within the muscle fibres and the greater uniformity of muscle fibre diameter. Gomori trichrome stain.

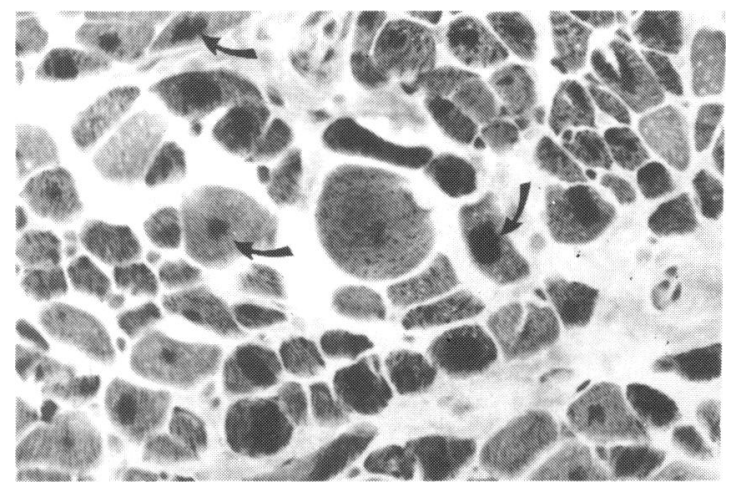

Fig. 8 Histological section of levator muscle from an affected side (MGP) showing clumping of the mitochondria in the centre of muscle fibres (arrowed). Note the wide variation in muscle fibre diameter. Gomori trichrome stain.

hospital to another and because it allowed a greater degree of freedom for the surgeon and laboratory.

The findings suggest that there is a loss of muscle fibres in the affected levator muscle relative to the clinically normal side. Furthermore, there is an impression that the levator muscles on both the affected and ostensibly normal sides in patients suffering the Marcus Gunn phenomenon contain fewer fibres than the equivalent area in the normal control levator muscles.

Morphometry of the muscle fibres suggests that there is both atrophy and compensatory hypertrophy of the remaining muscle fibres in the affected levator muscle in comparison with the clinically normal levator muscle and the normal control levator muscle. The presence of small fibres suggests that the atrophic process may be ongoing, but the relative absence of pyknotic muscle fibres and macrophages in the perimysium further suggests that the process may be in abeyance in some cases at the time of surgery. The impression from the morphology is of a weak muscle in a compensating or steady state.

The presence of muscle fibre type grouping with associated loss of fibres and atrophy and hypertrophy of the remaining muscle fibres suggests that the underlying process is a neurogenic atrophy. There appears to have been a partial loss of the nerve supply to areas of the muscle with reinnervation, resulting in a loss of the normal speckled pattern of type 1 and type 2 fibres. The underlying principle is that if, for example, in an area in which type 2 muscle fibres lose their nerve supply and are reinnervated by an adjacent sprouting nerve that has a type 1 pattern of electrical stimulation, then the previous type 2 muscle fibres undergo physiological and biochemical adaptation to become type 1 fibres as recognised by 
their histochemical staining reaction. This may result in focal areas assuming a monomorphic (rather than the normal speckled) type of fibre distribution dictated by the available nerve supply.

Examination of the clinically normal levator muscle in 10 of the 12 cases showed that they too contain fewer fibres than would be expected in a normal muscle in comparison with the normal control levator muscles. Two cases showed values consistent with normal fibre densities. Similarly, morphometry of the clinically normal levator muscle showed that there was a spread of muscle fibre diameters that was greater than that seen in the normal control levator muscles owing to the presence of both atrophic and hypertrophic forms. Fibre type grouping was present in some of the clinically normal muscles, though less obviously than seen in muscle from the affected side.

Both sides in the patients affected by the Marcus Gunn phenomenon showed central accumulations of mitochondria within scattered groups of individual fibres. This is not an appearance generally seen in normal skeletal or extrocular muscle but has been noted $^{14}$ is in biopsies of overacting inferior oblique muscles excised during the surgical treatment of strabismus. It was construed as being a reactive change to an increased work load or stress, but the rationale behind this change is uncertain.

Our findings suggest that the process underlying the Marcus Gunn phenomenon is a neurogenic atrophy with aberrant reinnervation and that it is a bilateral process with one side being severely affected and the clinically normal side being affected to a lesser degree. This may explain case reports of bilateral Marcus Gunn phenomenon. It would also indicate that the initiating pathological process was probably located within the central nervous system, that is, brain stem, rather than being a peripheral lesion of the third cranial nerve and that the initial lesion had occurred in utero.

\section{References}

1 Gunn RM. Congenital ptosis with peculiar associated movement of the affected lid. Trans Ophthalmol Soc UK 1883; 3: 283-7.

2 Hepler RS, Hoyt WF, Loeffler JD. Paradoxical synkinetic levator inhibition and excitation: an electromyographic study of unilateral oculopalpebral and bilateral mandibulo palpebral (Marcus Gunn) synkinesis in a 74-year-old man. Arch Neurol 1968; 18: 416-24.

3 Harman NB. The innervation of the orbicularis palepbrum muscle. Trans Ophthalmol Soc UK 1903; 23: 356-72.

4 Wartenberg R. Winking-jaw phenomenon. Arch Neurol Psychiatry 1948; 59: 734-53.

5 Berke RN, Wadsworth JAC. Histology of levator muscle in congenital and acquired ptosis. Arch Ophthalmol 1955; 53: 413-28.

6 Siemerling E. Anatomischer Befund bei einseitiger congenitaler Ptosis. Archiv für Psychiatrie und Nervenkrankheiten 1891-2; 23: 764-74.

7 Croker BP, Bosen EH, Brinn NT, Hammond FA. A fixative for use in muscle histochemistry. J Histochem Cytochem 1983; 31: $110-2$

8 Engel WK, Cunningham GG. Rapid examination of muscle tissue. Neurology 1963; 13: 919-23.

9 Brooke MH, Kaiser KK. Three 'myosin ATPase' systems: the nature of their $\mathrm{pH}$ lability and sulphydril dependence. $J$ Histochem Cytochem 1970; 18: 670-2.

10 Hoogenraad TU, Jennekens FGI, Tan KEWP. Histochemical fibre types in human extraocular muscles. An investigation of inferior oblique muscles. Acta Neuropathol (Berl) 1979; 45: 73-8.

11 Pearse AGE. Histochemistry: theoretical and applied. 3rd ed. London: Churchill-Livingstone; 1972: 2: 1342-3.

12 Buritone MS. Enzyme histochemistry and its application in the study of neoplasms. New York: Academic Press. 1962: 276.

13 Bancroft JD. Histochemical technique. London: Butterworths, 1967: 211-3

14 Spenser RF, McNeer KW. Structural alterations in overacting inferior oblique muscles. Arch Ophthalmol 1980; 98: 128-31.

15 Mayer E, Ludatscher RM, Zonis S. Primary and secondary overacting inferior oblique muscles: an ultrastructural study. $\mathrm{Br} J$ Ophthalmol 1984; 68: 416-20.

Accepted for publication 15 December 1986. 